\title{
Tratamientos farmacológicos para COVID-19: revisión sistemática viva y meta-análisis en red
}

\author{
Pharmacological treatments for COVID-19: living systematic review and network meta-analysis
}

\section{Comentado de:}

Siemieniuk RA, et al. BMJ. 2020 Jul 30;370:m2980. PMID: $32732190^{1}$

\section{Objetivo}

Comparar los efectos de los tratamientos para la enfermedad por coronavirus 2019 (COVID-19).

\section{Diseño}

Revisión sistemática viva (RSV) y meta-análisis en red (MAR). Su protocolo no fue registrado, se incluye como complemento de la publicación.

Esta RSV se actualizará para reflejar la evidencia emergente. Las actualizaciones pueden ocurrir hasta por dos años a partir de la fecha de publicación original.

\section{Fuentes de datos}

Búsquedas diarias en la base de datos descargable de artículos de investigación de COVID-19 del Centro para el Control y la Prevención de Enfermedades (CDC, por sus iniciales en inglés) de los EE.UU., que incluye 25 bases de datos electrónicas y seis bases de datos chinas hasta el 20 de julio de 2020. Para identificar ensayos clínicos aleatorizados (ECA), se utilizó un modelo de aprendizaje automático validado y altamente sensible ${ }^{2}$.

\section{Selección de estudios}

Se incluyeron ECA realizados con personas con COVID-19 sospechoso, probable o confirmado, aleatorizadas a tratamiento farmacológico, atención estándar o placebo. Se excluyeron estudios sobre vacunación, hemoderivados, nutrición y hierbas tradicionales chinas compuestas. Los artículos potencialmente elegibles fueron examinados por pares de revisores en forma indepen- diente.

\section{Métodos}

Después de la extracción de datos por duplicado se realizó un MAR de efectos aleatorios bayesianos. El riesgo de sesgo de los estudios incluidos se evaluó mediante una modificación de la herramienta Cochrane de riesgo de sesgo 2.0. Los resultados de interés se seleccionaron en función de su importancia para los pacientes mediante un panel de pacientes y expertos clínicos con representación global y sin conflicto de intereses. Para cada resultado, las intervenciones se clasificaron en grupos, de más a menos beneficiosas o perjudiciales, siguiendo el abordaje GRADE para calificar la confianza en la evidencia y resumir los hallazgos.

\section{Resultados}

Se incluyeron 23 ECA. La confianza en la evidencia para la mayoría de las comparaciones fue muy baja debido al riesgo de sesgo (por falta de cegamiento) y a la imprecisión sustancial. Los resultados principales para los corticoides, la hidroxicloroquina la combinación lopinavir-ritonavir, el remdesivir y el umifenovir se resumen en la Figura 1. Otras intervenciones en evaluación, para las que no se pudieron realizar estimaciones debido a la falta de datos, la escasa cantidad de participantes o la baja ocurrencia de eventos, incluyeron: el ácido alfa-lipoico, el baloxavir marboxil, la cloroquina (fármaco que se trató como un nodo separado para los eventos adversos y se combinó con hidroxicloroquina para todos los demás desenlaces), el glicirrizinato de diamonio, el favipiravir, el interferón beta-1a, el novaferon, la combinación de novaferon y lopinavir-ritonavir, la ribavirina, la combinaciones ribavirinainterferón beta-1b y ribavirina-lopinavir-ritonavir, y el ruxolitinib.

Figura 1. Resumen de efectos de las intervenciones para el tratamiento de pacientes con COVID-19 vs. cuidados usuales.

\begin{tabular}{|c|c|c|c|c|c|c|c|c|}
\hline & $\begin{array}{c}\text { Mortalidad a } \\
90 \text { días } \\
\text { DRA (ICr } 95 \%)\end{array}$ & $\begin{array}{l}\text { VM a } 90 \text { días } \\
\text { DRA (ICr 95\%) }\end{array}$ & $\begin{array}{c}\text { Eventos } \\
\text { adversos } \\
\text { DRA (ICr 95\%) }\end{array}$ & $\begin{array}{l}\text { Eliminación } \\
\text { viral a } 7 \text { días } \\
\text { DRA (ICr 95\%) }\end{array}$ & $\begin{array}{c}\text { Estadía } \\
\text { hospitalaria } \\
\text { DM (ICr 95\%) }\end{array}$ & $\begin{array}{c}\text { Duración } \\
\text { de VM } \\
\text { DM (ICr 95\%) }\end{array}$ & $\begin{array}{l}\text { Resolución } \\
\text { de síntomas } \\
\mathrm{DM}(\mathrm{ICr} 95 \%)\end{array}$ & $\begin{array}{c}\text { Eliminación } \\
\text { viral (tiempo) } \\
\text { DM (ICr 95\%) }\end{array}$ \\
\hline Cuidados usuales $^{ \pm}$ & $330 / 1000$ & $116 / 1000$ & $15 / 1000$ & $500 / 1000$ & 7 días & 10 días & 19 días & 8 días \\
\hline Corticoides & $\begin{array}{c}-37 \\
(-63 a-11) \dagger\end{array}$ & $\begin{array}{c}-31 \\
(-47 a-9) \dagger\end{array}$ & & & & & & \\
\hline Hidroxicloroquina & $\begin{array}{c}0 \\
(-330 \text { a } 670)\end{array}$ & & $\begin{array}{c}985 \\
\text { (25 a } 985)\end{array}$ & $\begin{array}{c}83 \\
(-343 \text { a } 414)\end{array}$ & & & $\begin{array}{c}-4,5 \\
(-6 a-3)\end{array}$ & $\begin{array}{c}-0,5 \\
(-3 \text { a } 3,5)\end{array}$ \\
\hline Lopinavir-ritonavir & $\begin{array}{c}-71 \\
(-197 \text { a 109) }\end{array}$ & & & $\begin{array}{c}-239 \\
(-478 \text { a } 235)\end{array}$ & $\begin{array}{c}-1,42(-3,03 \\
\text { a } 0,02)\end{array}$ & & $\begin{array}{c}-1,2 \\
(-2 a-0,4)\end{array}$ & $\begin{array}{c}-0,2 \\
(-3,1 \mathrm{a} 4,2)\end{array}$ \\
\hline Remdesivir & $\begin{array}{c}-85 \\
(-164 \text { a 29) }\end{array}$ & $\begin{array}{c}-24 \\
(-70 \text { a } 52)\end{array}$ & $\begin{array}{c}4 \\
(-7 a 42) \\
\end{array}$ & $\begin{array}{c}11 \\
(-469 a \mathrm{a} 472)\end{array}$ & $\begin{array}{c}0,4 \\
(-3,8 \mathrm{a} 4,5)\end{array}$ & $\begin{array}{c}-5,3 \\
(-15,2 \text { a } 5)\end{array}$ & $\begin{array}{c}-2,6 \\
(-4,3 \text { a }-0,5)\end{array}$ & \\
\hline Umifenovir & $\begin{array}{c}0 \\
(-330 \text { a } 670)\end{array}$ & & & & & & & \\
\hline
\end{tabular}

${ }^{ \pm}$Para los desenlaces mortalidad y ventilación mecánica se utilizó la base de datos internacional de infecciones respiratorias agudas graves e infecciones emergentes COVID-19; para todos los demás resultados, la mediana de los estudios en los que los participantes recibieron atención estándar.

+ La mejor estimación del efecto se obtuvo a partir de evidencia directa. VM: ventilación mecánica; ICr: intervalo de credibilidad

\begin{tabular}{l|l|l|l|l|r|}
\multicolumn{1}{l}{ Certeza } & \multicolumn{2}{c}{ Mayor beneficio } & \multicolumn{2}{c}{ Beneficio intermedio } & \multicolumn{2}{c}{ Perjudicial } & \multicolumn{1}{c}{ Datos insuficientes } \\
Alta/moderada & & & & & $<100$ pacientes \\
\cline { 2 - 5 } Baja / muy baja & & & & & Sin datos \\
\cline { 2 - 6 }
\end{tabular}


Los corticoides fueron la única intervención con evidencia de reducción de la mortalidad en comparación con la atención estándar (37 muertes menos cada 1000 pacientes, intervalo de credibilidad [ $\mathrm{ICr}$ ] del 95\%: 11 a 63 menos, certeza moderada) y la ventilación mecánica (31 pacientes ventilados menos por 1000 pacientes, ICr $95 \% 9$ a 47 menos, certeza moderada); ambas estimaciones estuvieron basadas en evidencia directa. Las estimaciones de la red para los corticoides en comparación con la atención estándar fueron menos precisas debido a su heterogeneidad.

Tres fármacos podrían reducir la duración de los síntomas en comparación con la atención estándar: la hidroxicloroquina (diferencia de medias [DM] -4,5 días, certeza baja), el remdesivir (DM -2,6 días, certeza moderada) y la combinación lopinavir-ritonavir (DM -1,2 días, certeza baja).

La hidroxicloroquina podría aumentar el riesgo de eventos adversos en comparación con las otras intervenciones, y es probable que el remdesivir no aumente sustancialmente el riesgo de efectos adversos que lleven a la suspensión del fármaco. Ninguna otra intervención incluyó suficientes pacientes para interpretar de manera concluyente los efectos adversos que llevaron a la interrupción del fármaco.

\section{Conclusión}

Los corticoides probablemente reducen la mortalidad y la ventilación mecánica en pacientes con COVID-19 en comparación con la atención estándar. La efectividad de la mayoría de las intervenciones es incierta, porque la mayoría de los ECA hasta ahora han sido pequeños y tienen limitaciones importantes.

Fuente de financiamiento/Conflicto de interés de los autores: Este estudio fue financiado por el Instituto de Investigación Sanitaria Canadiense (CIHR-IRSC:0579001321). Todos los autores declararon no tener conflictos de interés que pudieran influenciar este trabajo.

\section{Comentario}

Al 9 de septiembre de 2020, más de 27 millones de personas han sido infectadas con el SARS-CoV-2, responsable de la COVID-19, y 898.000 personas han muerto ${ }^{3}$. Con más de 1.800 ensayos completados o en curso, la evidencia de un tratamiento efectivo para esta enfermedad sigue siendo de muy baja calidad. Sin embargo, ante la presión que ejerce la pandemia, los fármacos señalados como terapias potenciales son indicados y administrados.

Ante una fuerte demanda de evidencia en un contexto de constante cambio de recomendaciones, las RSV, por su síntesis acumulativa de actualización periódica, y los MAR, por aprovechar toda la red de evidencia y su capacidad de generar comparaciones indirectas y "rankings" terapéuticos ${ }^{4}$, pueden proporcionar el más completo panorama general de la evidencia relevante en un momento específico. El presente estudio es parte del proyecto BMJ Rapid Recommendations ${ }^{5}$, un esfuerzo colaborativo entre MAGIC Evidence Ecosystem Foundation ${ }^{6}$ y The BMJ que intenta dar cuenta de esta demanda.

El estudio resumido mostró que la única intervención que probablemente reduzca la mortalidad y la necesidad de ventilación mecánica sean los corticoides, conclusión completamente derivada de los resultados del ensayo RECOVERY ${ }^{7,8}$.

El remdesivir es la única intervención en la que existe una certeza moderada de evidencia -sólo proveniente de ECA patrocinados por una empresa farmacéutica- que respalda los beneficios en el tiempo a la resolución de los síntomas, pero sigue siendo incierto si el remdesivir tiene algún efecto sobre la mortalidad u otros resultados importantes para los pacientes.

Lamentablemente, la evidencia directa proveniente de ECA en pacientes con COVID-19 ha proporcionado, hasta ahora, poca evidencia definitiva sobre los efectos adversos de la mayoría de las intervenciones.

La hidroxicloroquina podría aumentar el riesgo de eventos adversos que lleven a la interrupción del fármaco en comparación con las otras intervenciones. El estudio RECOVERY, el mayor ECA sobre hidroxicloroquina, sugiere que la hidroxicloroquina podría no reducir la mortalidad y podría aumentar la duración de la estadía hospitalaria ${ }^{9,10}$, aunque en la próxima actualización -que incluirá cuatro ECA adicionales- esto podría modificarse. No se encontraron pruebas convincentes de que las otras intervenciones produjeran beneficios o daños en comparación con la atención estándar.

Entre las fortalezas de este estudio se destacan la exhaustividad y actualización de la búsqueda, el equipo compuesto por expertos clínicos, metodólogos y pacientes, el uso del enfoque GRADE, la comunicación mediante infografía interactiva y la disponibilidad de un sitio web para que los usuarios accedan a los resultados más actualizados. Como debilidades observamos que muchos resultados no fueron informativos y, a veces, no fueron plausibles, por lo que sólo se reportaron los resultados de los tratamientos para aquellos desenlaces e intervenciones en los que se aleatorizaron al menos 100 personas o se produjeron 20 eventos. La principal limitación de la revisión sistemática es la muy baja confianza en la evidencia como resultado de la escasez de datos actualmente disponibles, la frecuente falta de cegamiento en los estudios primarios, incluso en los ensayos más grandes, y la baja calidad del reporte. Además un volumen considerable de evidencia quedó, de momento, fuera del análisis. Un cuestionamiento realizado tras la publicación del artículo fue que utilizar un código de color indicando beneficio en casos en que la confianza o certeza en la evidencia es muy baja podría resultar engañoso, por lo que resulta conveniente ser muy prudente al realizar cualquier inferencia en estos casos.

Los ensayos exclusivamente patrocinados por la industria, como los de remdesivir y otros medicamentos tutelados por patentes, podrían estar particularmente expuestos al riesgo de sesgo de publicación y requerir una interpretación más cautelosa que los medicamentos probados en ECA independientes. La inclusión de prepublicaciones minimiza este riesgo, pero al mismo tiempo podría introducir sesgos por errores e informes de baja calidad.

Por otro lado, es posible que los grupos de atención estándar no cegados recibieran co-intervenciones sistemáticamente diferentes a las de los grupos aleatorizados a recibir placebo, y por esta razón se disminuyó la confianza en la evidencia derivada de las comparaciones directas dominadas por estudios no cegados, según el abordaje GRADE. Esta RSV y MAR utiliza un enfoque mínimamente contextualizado para calificar la certeza de la evidencia, mientras que para BMJ Rapid Recommendations se usa un enfoque completamente contextualizado, en el que los umbrales de importancia de las magnitudes de los 
efectos dependen de todos los demás resultados y de otros factores que intervienen en la decisión. Los resultados pueden ser potencialmente engañosos cuando la red es escasa, casos en los cuales conviene centrarse en estimaciones directas de estudios más grandes, lo que explica las diferencias en los detalles de las estimaciones del efecto en este MAR.

Hasta la fecha, existen otros tres esfuerzos similares para intentar resumir la evidencia disponible sobre los tratamientos para la COVID-19 ${ }^{11-13}$. Aunque existan razones prácticas o metodológicas para proceder de forma independiente, incluyendo la replicabilidad de los resultados utilizando diferentes enfoques científicos, lo cierto es que lo ideal sería crear redes colaborativas tanto en investigación primaria (p. ej., con la vacuna para COVID-19) como en investigación secundaria de síntesis de evidencia para optimizar la eficiencia de los recursos disponibles a nivel global.

\section{Conclusiones del comentador}

La evidencia de esta RSV y MAR sugiere que los corticoides probablemente reduzcan la mortalidad y la necesidad de ventilación mecánica en pacientes con COVID-19 grave. El remdesivir y la combinación lopinavir-ritonavir pueden reducir la duración de los síntomas; aunque la hidroxicloroquina también podría hacerlo, ésta incrementa significativamente los efectos adversos.

Para otros desenlaces y para el resto de las intervenciones farmacológicas los resultados son actualmente muy inciertos y no existe evidencia concluyente sobre beneficios o daños importantes. Probablemente las nuevas rondas de actualización mejoren la precisión y la certeza de las estimaciones. Ante este panorama, en el que todavía reina la incertidumbre, tienen un rol fundamental la toma de decisiones compartidas, a nivel clínico, y la cautela, a nivel sanitario.

Agustín Ciapponi [ Servicio de Medicina Familiar y Comunitaria, Hospital Italiano de Buenos Aires; Centro Cochrane Argentina, Instituto de Efectividad Clínica y Sanitaria. aciapponi@gmail.com.ar ]

Ciapponi A. Tratamientos farmacológicos para COVID-19: revisión sistemática viva y meta-análisis en red. Evid Actual Pract Ambul. 2020;23(3):e002092. Comentado de: Siemieniuk RA, et al. Drug treatments for covid-19: living systematic review and network meta-analysis. BMJ. 2020 Jul 30;370:m2980. PMID: 32732190

\section{Referencias}

1. Siemieniuk RA, Bartoszko JJ, Ge L, et al. Drug treatments for covid-19: living systematic review and network meta-analysis. BMJ. 2020;370:m2980. Available from: 10.1136/bmj.m2980.

2. Marshall IJ, Noel-Storr A, Kuiper J, et al. Machine learning for identifying Randomized Controlled Trials: An evaluation and practitioner's guide. Res Synth Methods. 2018;9(4):602-614. Available from: 10.1002/jrsm.1287.

3. COVID-19 Dashboard by the Center for Systems Science and Engineering at Johns Hopkins University; 2020. Available from: https://gisanddata. maps.arcgis.com/apps/opsdashboard/index.html\#/bda7594740fd40299423467b48e9ecf6 [Last access: 2020-09-09].

4. Ciapponi A. La ola de los meta-análisis en red: una herramienta cada vez más útil para la toma de decisiones. Evid Act Pract Ambul. 2018;21(4):9899.

5. Siemieniuk RA, Agoritsas T, Macdonald H, et al. Introduction to BMJ Rapid Recommendations. BMJ. 2016;354:i5191. Available from: 10.1136/bmj. i5191.

6. MAGIC (Making GRADE the Irresistible Choice) Evidence Ecosystem Foundation; 2018. Available from: http://magicproject.org/ [Last access: 202009-09].

7. Horby P, Lim WS, Emberson J, RECOVERY Collaborative Group, et al. Effect of Dexamethasone in Hospitalized Patients with COVID-19: Preliminary Report. N Engl J Med. 2020;EJMoa2021436. Available from: 10.1056/NEJMoa2021436.

8. Ciapponi A. La dexametasona redujo la mortalidad de pacientes COVID-19 en ventilación mecánica invasiva u oxigenoterapia. Comentado de: Horby P, et al. Effect of Dexamethasone in Hospitalized Patients with COVID-19 - Preliminary Report. medRxiv2020. doi:10.1101/2020.06.22.20137273. Evid Actual Pract Ambul. 2020;23(3):2078-2078.

9. Horby P, Mafham M, Linsell L. Effect of Hydroxychloroquine in Hospitalized Patients with COVID-19: Preliminary results from a multi-centre, randomized, controlled trial.; 2020. Available from: https://doi.org/10.1101/2020.07.15.20151852;https://www.medrxiv.org/content/10.1101/2020.07.15. 20151852v1\#: :text=Conclusions\%3A\%20In\%20patients\%20hospitalized\%20with,invasive\%20mechanical\%20ventilation\%20or\%20death.

10. Ciapponi A. El escándalo de la cloroquina. Evid Act Pract Ambul. 2020;23(3):2073-2073.

11. Pan American Health Organization. Ongoing Living Update of Potential COVID-19 Therapeutics: Summary of Rapid Systematic Reviews; 2020. Available from: https://iris.paho.org/handle/10665.2/52294 [Last access: 2020-09-09]

12. Juul S, Nielsen N, Bentzer P, et al. Interventions for treatment of COVID-19: a protocol for a living systematic review with network meta-analysis including individual patient data (The LIVING Project). Syst Rev. 2020;9(1):108-108. Available from: 10.1186/s13643-020-01371-0;https://dx.doi. org/10.1186/s13643-020-01371-0.

13. Boutron I, Chaimani A, Devane D, et al.. Interventions for preventing and treating COVID-19: protocol for a living mapping of research and a living systematic review; 2020. Available from: 10.5281/zenodo.3820266. 